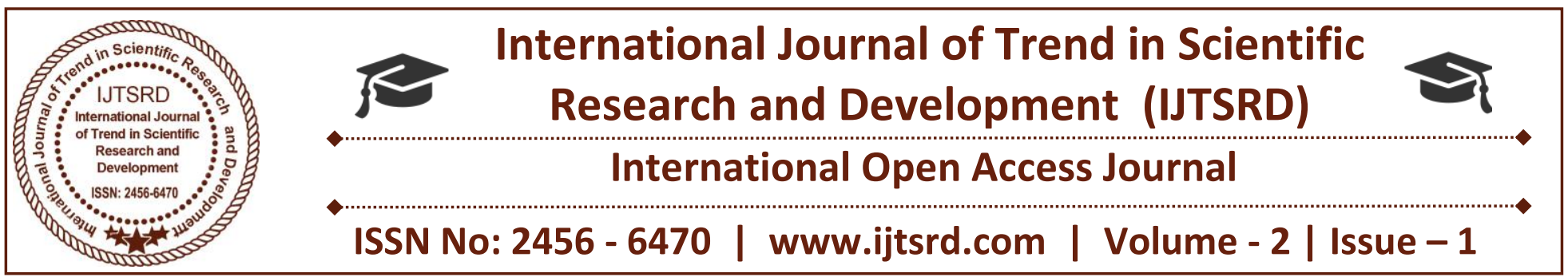

\title{
Performance Study on Multipath Routing Algorithm using Temporarily Ordered Routing Algorithm (TORA) using Link - Reversal in Wireless Networks
}

\author{
Dr. K. Kumaravel \\ Professor \& Head, Department of Computer Science, \\ Dr.N.G.P. Arts \& Science College, Coimbatore
}

\author{
Dr. M. Sengaliappan \\ Prof \& Dean, Department of Computer Science, \\ Kovai Kalaimaghal Arts \& Science College, \\ Coimbatore
}

\section{ABSTRACT}

We present new distributed routing protocol Multipath Routing wireless networks. The protocol is one of a family of protocols which we term "link reversal" algorithms. The protocol's reaction is structured as a temporally-ordered sequence of diffusing computations; each computation consisting of a sequence of directed link reversals. The protocol is highly adaptive, efficient and scalable; being bestsuited for use in large, dense, mobile networks. In these networks, the protocol's reaction to link failures typically involves only a localized "single pass" of the distributed algorithm. This capability is unique among protocols which are stable in the face of network partitions, and results in the protocol's high degree of adaptively. This desirable behavior is achieved through the novel use of a "physical or logical clock" to establish the "temporal order" of topological change events which is used to structure (or order) the algorithm's reaction to topological changes. We refer to the protocol as the temporally-ordered routing algorithm (TORA).

Keywords: TORA, LLS, DSM, DSR, LINK REVERSAL ALGORITHM (LRA)

\section{INTRODUCTION}

In Wireless Networks the Routing is mainly considered with Multi-path Dynamic routing with leave and join group of heterogeneous nodes based on Energy, Time on Arrival(TOA), Bandwidth, Link reliability. The Temporally-Ordered Routing Algorithm (TORA) [1] is a distributed routing algorithm for mobile, multipath, wireless networks which builds upon the earlier work of [2] and [3]. It is best suited for use in large, dynamic, bandwidthconstrained networks such as those proposed for future mobile military systems. TORA is designed to minimize reaction to topological changes. A key concept in its design is that it largely decouples the generation of potentially far-reaching control message propagation from the rate of topological changes. Control messaging is typically localized to a very small set of nodes near the change without having to resort to a dynamic, hierarchical routing solution with its attendant complexity. This localization is achieved at the cost (or benefit?) of not performing a shortestpath routing computation-i.e., TORA does not perform shortest-path routing. However, for the conditions expected in large mobile networks, it will be seen that this approach is superior to link-state routing. This paper presents a detailed performance comparison of TORA with Ideal Link-State (ILS) routing and pure flooding [4]. Comparison with ILS is useful due to its simplicity and familiarity. Furthermore, ILS technology is the basis for the Open Shortest Path First (OSPF) [5] routing protocol currently under consideration for use by the U.S. military in its large mobile networks. Comparison with flooding is secondary, and is useful to see the network environment in which a more efficient routing technique than flooding is necessary. 
Comparison with flooding is also useful since one might expect that — as the rate of topological change increases in a dynamic network, eventually all other routing algorithms will essentially "breakdown" leaving flooding as the only recourse. It is useful to know whether our test scenarios are operating at or near this breakdown point. The paper is organized as follows: a brief description of TORA is given in section 2, a detailed description of the simulation design is given in section 3, the performance results for the scenarios we considered are given in section 4, and some final thoughts and future work are given in section 5 .

\section{OBJECTIVES AND MOTIVATION OF THE RESEARCH}

Multi-path routing is a routing technique that enables data transmission over multiple paths, is an effective strategy in achieving reliability in wireless mesh networks. The scope of this research work is to develop hybrid multi-path routing protocol based on Hybrid Swarm Intelligence algorithm. This work investigates the routing metrics advantages and disadvantages. Based on the routing metrics the hybrid multi-path routing protocol is developed.

To improve the multi-path routing protocol and path selection process in wireless mesh network using novel algorithms.

To develop a novel model to reduce the energy consumption in the network by selecting optimal path in the wireless network.
To analyze the topological changes may occur in industrial fields due to the faulty nodes or imminent external environmental factors in wireless mesh networks.

To experiment the multipath the factors that bandwidth, packet delivery ratio, and expected Transmission Time.

To develop an efficient greedy based algorithms to estimate the bandwidth of the network to avoid delay and time consumption of network. Temporally Ordered Routing Algorithm (TORA) is a highly adaptive, efficient and scalable distributed routing algorithm based on the concept of link reversal [3]. TORA is proposed for highly dynamic mobile, multihop wireless networks. It is a source-initiated ondemand routing Protocol. It finds multiple routes from a source node to a destination node. The main feature of TORA is that the control messages are localized to a very small set of nodes near the occurrence of a topological change. To achieve this, the nodes maintain routing information about adjacent nodes. The protocol has three basic functions: Route creation Route maintenance and Route erasure . TORA can suffer from unbounded worst-case convergence time for very stressful scenarios [15,17]. TORA has a unique feature of maintaining multiple routes to the destination so that topological changes do not require any reaction at all. The protocol reacts only when all routes to the destination are lost. In the event of network partitions the protocol is able to detect the partition and erase all invalid routes. Table 1 lists some comparisons between the three routing protocols discussed above.

\begin{tabular}{|c|c|c|c|}
\hline Parameters & AODV & DSR & TORA \\
\hline $\begin{array}{l}\text { Source } \\
\text { Routing }\end{array}$ & No & Yes & No \\
\hline Topology & Ful1 & Fu11 & Reduced \\
\hline Broadcast & Full & Full & Local \\
\hline $\begin{array}{c}\text { Update } \\
\text { information }\end{array}$ & $\begin{array}{l}\text { Route } \\
\text { error }\end{array}$ & $\begin{array}{l}\text { Route } \\
\text { error }\end{array}$ & $\begin{array}{l}\text { Node"s } \\
\text { height }\end{array}$ \\
\hline $\begin{array}{c}\text { Update } \\
\text { destination }\end{array}$ & Source & Source & Neighbors \\
\hline Method & Unicast & Unicast & Broadcast \\
\hline $\begin{array}{c}\text { Storage } \\
\text { Complexity }\end{array}$ & $O(E)$ & $O(E)$ & $O\left(D d^{*} A\right)$ \\
\hline
\end{tabular}

\section{LINK REVERSAL ALGORITHM}

Link reversal is a versatile algorithm design technique that has been used in numerous distributed algorithms for a variety of problems. The common thread in these algorithms is that the distributed system is viewed as a graph, with vertices representing the computing nodes and edges representing some other feature of the system (for instance, point-to-point communication channels or a conflict relationship). Each algorithm 
assigns a virtual direction to the edges of the graph, producing a directed version of the original graph. As the algorithm proceeds, the virtual directions of some of the links in the graph change in order to accomplish some algorithm-specific goal. The criterion for changing link directions is based on information that is local to a node (such as the node having no outgoing links) and thus this approach scales well, a feature that is desirable for distributed algorithms.

This Research presents, in a tutorial way, a representative sampling of the work on link-reversalbased distributed algorithms. The algorithms considered solve routing, leader election, mutual exclusion, distributed queuing, scheduling, and resource allocation. The algorithms can be roughly divided into two types, those that assume a more abstract graph model of the networks, and those that take into account more realistic details of the system. In particular, these more realistic details include the communication between nodes, which may be through asynchronous message passing, and possible changes in the graph, for instance, due to movement of the nodes.

Therefore, there are new challenges for routing protocols in wireless Networks using Sensor\& Signal based on the transmitting and Receiving the signal in Radio waves. The routing can be measured the Bandwidth which includes Energy signals based performance may use the Routing Algorithm to overcome the delay convergence of TORA to improve the Packet Delivery Fraction. Since traditional routing protocols may not be suitable for MANETs. Researchers are designing new routing protocols, comparing and improving existing routing protocols before any routing protocols are standardized using simulations. This work is an attempt towards a comprehensive performance evaluation of three commonly used hoc routing protocols (DSR, TORA and AODV). Over the past few years, new standards have been introduced to enhance the capabilities of ad hoc routing protocols. As a result, ad hoc networking has been receiving much attention from the wireless research community.

\section{REFERENCES:}

1) V. Park and M. S. Corson, "A Highly Adaptive Distributed Routing Algorithm for Mobile Wireless Networks", Proc. IEEE INFOCOM '97, Kobe, Japan (1997).
2) E. Gafni and D. Bertsekas, "Distributed Algorithms for Generating Loop-free Routes in Networks with Frequently Changing Topology," IEEE Trans. Commun. (January 1981).

3) M. S. Corson and A. Ephremides, "A Distributed Routing Algorithm for Mobile Wireless Networks," Wireless Networks 1 (1995).

4) D. Bertsekas and R. Gallager, Data Networks (Prentice-Hall, 1987).

5) J. Moy, “OSPF Version 2," Internet RFC 1583, Proteon, Inc., March 1994.

6) P. Merlin and A. Segall, "A Failsafe Distributed Routing Protocol," IEEE Trans. Commun. (September 1979).

7) J. Jaffe and F. Moss, "A Responsive Distributed Routing Algorithm for Computer Networks,"

IEEE Trans. Commun. (July 1982).

8) J. J. Garcia-Luna-Aceves, "Loop-free Routing Using Diffusing Computations," IEEE Trans. Networking 1(1) (1993).

9) C. Perkins and P. Bhagwat, "Highly Dynamic Destination-Sequenced Distance Vector Routing (DSDV) for Mobile Computers," ACM SIGCOMM , (October 1994).

10) P. Humblet, "Another Adaptive Shortest-Path Algorithm," IEEE Trans. Commun. (June 1991).

11) Samir R. Das, Charles E. Perkins, Elizabeth E. Royer, "Performance Comparison of Two Ondemand Routing Protocols for Ad Hoc Networks".

12) Johansson, P., T. Larsson, N. Hedman, B. Mielczarek, and M. Degermark. 1999. Scenariobased performance analysis of routing protocols for mobile ad-hoc networks. Paper presented at Mobicom'99, August, Seattle, WA.

13) Samir R. Das, Robert Castaneda and Jiangtao Ya $\mathrm{n}$, "Simulation- based performance evaluation of rout ing protocols for mobile ad hoc networks".

14) Samba Sesay, Zongkai Yang, Biao Qi and Jianhua He, "Simulation comparison of Four Wireless Ad Hoc Routing Protocols".

15) Park V. and S. Corson, 2001. Temporary-ordered Routing Algorithm (TORA). Internet Draft, draf tietf-manettora-spec-04.txt.

16) C. Perkins, Ad hoc On demand Distance Vector (AODV) routing, IETF Internet draft (1997), 
http:// www.ietf.org/intern et-drafts/draft- ietfmanet-aodv-00.txt.

17) V. Park and S. Corson, Temporally Ordered Routing Algorithm (TORA) Version 1, Functional specification IETF Internet draft (1998), http://www.ietf.org/internet-drafts/draft-ietfmanet-tora-spec-01.txt.

18) E. M. Royer and C. Toh, "A Review of Current Routing Protocols for Ad Hoc Mobile Wireless Networks," IEEE Personal Communications , pp. 46-55, Apr

\section{AUTHORS}

1) Dr M.Sengaliappan, working as a Professor \& Dean, Dept of Computer Science, KKCAS College, Coimbatore. He has more than two decades of Experience in Teaching \& Research.

2) Dr.K.Kumaravel, working as a Professor \& Head , Dept of Computer Science, Dr.N.G.P. Arts \& Science College, Coimbatore. He has more than two decades of Experience in Teaching \& Research 